Mathematical Models and Methods in Applied Sciences

Vol. 24, No. 14 (2014) 2943-2948

(C) World Scientific Publishing Company

DOI: $10.1142 / \mathrm{S} 0218202514990012$

\title{
Author index \\ Volume 24
}

Abert, C., see Exl

Aki, G. L., Dreyer, W., Giesselmann, J. \& Kraus, C., A quasi-incompressible diffuse interface model with phase transition

Alicandro, R. \& Ansini, N., A variational model of interaction between continuum and discrete systems

Allaire, G. \& Dapogny, C., A linearized approach to worst-case design in parametric and geometric shape optimization

Almeida, R. C., see Lima

Amaziane, B., Pankratov, L. \& Piatnitski, A., Homogenization of immiscible compressible two-phase flow in highly heterogeneous porous media with discontinuous capillary pressures

Amendola, G., Fabrizio, M. \& Golden, J. M., Free energies in a general nonlocal theory of a material with memory

Andreianov, B., Donadello, C. \& Rosini, M. D., Crowd dynamics and conservation laws with nonlocal constraints and capacity drop

Ansini, N., see Alicandro

Antonietti, P. F., Beirão da Veiga, L., Bigoni, N. \& Verani, M., Mimetic finite differences for nonlinear and control problems

Arnold, D. N., Awanou, G. \& Winther, R., Nonconforming tetrahedral mixed finite elements for elasticity
24 (2014) 1877

24 (2014) 1957

24 (2014) 2199

24 (2014) 2569

24 (2014) 1421

24 (2014) 1037

24 (2014) 2685

24 (2014) 1957

24 (2014) 1457

24 (2014) 783
Aurada, M., Melenk, J. M. \& Praetorius, D., Mixed conforming elements for the large-body limit in micromagnetics

Awanou, G., see Arnold

Ball, J. M. \& Koumatos, K., An investigation of non-planar austenitemartensite interfaces

Barrett, J. W. \& Prigozhin, L., Existence and approximation of a mixed formulation for thin film magnetization problems in superconductivity

Bassi, F., Botti, L. \& Colombo, A., Agglomeration-based physical frame $d G$ discretizations: An attempt to be mesh free

Bazilevs, Y., see Takizawa

Beirão da Veiga, L., Brezzi, F., Marini, L. D. \& Russo, A., The Hitchhiker's Guide to the Virtual Element Method

Beirão da Veiga, L., see Antonietti

Bellettini, G., Chambolle, A. \& Goldman, M., The $\Gamma$-limit for singularly perturbed functionals of Perona-Malik type in arbitrary dimension

Bellomo, N. \& Brezzi, F., Complex systems: New challenges with modeling headaches

Bellomo, N., Brezzi, F. \& Manzini, G., Recent techniques for PDE discretizations on polyhedral meshes

Bendahmane, M., Khalil, Z. \& Saad, M., Convergence
24 (2014) 113

24 (2014) 783

24 (2014) 1937

24 (2014) 991

24 (2014) 1495

24 (2014) 2437

24 (2014) 1541

24 (2014) 1457

24 (2014) 1091

24 (2014) 213

24 (2014) 1453 
of a finite volume scheme for gas-water flow in a multi-dimensional porous medium

Bergounioux, M., Bonnefond, X., Haberkorn, T. \& Privat, Y., An optimal control problem in photoacoustic tomography

Berninger, H., Ohlberger, M., Sander, O. \& Smetana, K., Unsaturated subsurface flow with surface water and nonlinear in- and outflow conditions

Bigoni, N., see Antonietti

Bissell, J. J., Caiado, C. C. S., Goldstein, M. \& Straughan, B., Compartmental modelling of social dynamics with generalised peer incidence

Bonnard, B. \& Cots, O., Geometric numerical methods and results in the contrast imaging problem in nuclear magnetic resonance

Bonnefond, X., see Bergounioux

Bonnivard, M., Dalibard, A.-L. \& Gérard-Varet, D., Computation of the effective slip of rough hydrophobic surfaces via homogenization

Borsche, R., Göttlich, S., Klar, A. \& Schillen, P., The scalar Keller-Segel model on networks

Borsche, R., Klar, A., Kühn, S. \& Meurer, A., Coupling traffic flow networks to pedestrian motion

Botti, L., see Bassi

Bouin, E., Calvez, V. \& Nadin, G., Hyperbolic traveling waves driven by growth

Bourgault, Y., see Coudière

Bouyssier, J., Pierre, C. \& Plouraboué, F., Mathematical analysis of parallel convective exchangers with general lateral boundary conditions using generalized Graetz modes
Boyer, F. \& Minjeaud, S., Hierarchy of consistent $n$ component Cahn-Hilliard

24 (2014) 2525

24 (2014) 901

24 (2014) 1457

24 (2014) 719

24 (2014) 187

24 (2014) 2525

24 (2014) 2259

24 (2014) 221

24 (2014) 359

24 (2014) 1495

24 (2014) 1165

24 (2014) 1115

24 (2014) 627 systems

Brezzi, F., see Beirão da Veiga

Brezzi, F., see Bellomo

Brezzi, F., see Bellomo

Bruckner, F., Suess, D., Feischl, M., Führer, T. Goldenits, P., Page, M., Praetorius, D. \& Ruggeri, M., Multiscale modeling in micromagnetics: Existence of solutions and numerical integration

24

(2014) 2885

24 (2014) 1541

24 (2014) 213

24 (2014) 1453

24 (2014) 2627

Buono, P.-L. \& Eftimie, R., Analysis of Hopf/Hopf bifurcations in nonlocal hyperbolic models for selforganised aggregations

Burman, E., Robust error estimates in weak norms for advection dominated transport problems with rough data

Caiado, C. C. S., see Bissell

Calo, V. M., Efendiev, Y. \& Galvis, J., Asymptotic expansions for high-contrast elliptic equations

Calvez, V., see Bouin

Calvo, J., Nieto, J., Soler, J. \& Vásquez, M. O., On a dispersive model for the unzipping of doublestranded DNA molecules

Cangiani, A., Georgoulis, E. H. \& Houston, P., $h p$-Version discontinuous Galerkin methods on polygonal and polyhedral meshes

Carrillo, J. A., Gwiazda, P. \& Ulikowska, A., Splitting-particle methods for structured population models: Convergence and applications

Casado-Díaz, J., CouceCalvo, J., Maestre, F. \& Martín Gómez, J. D., Homogenization and correctors for the wave equation with periodic coefficients

24 (2014) 2663

24 (2014) 719

24 (2014) 465

24 (2014) 1165

24 (2014) 495

24 (2014) 2009

24 (2014) 2171

Challa, D. P., Hu, G. \& Sini, M., Multiple scattering of
24 (2014) 1343 
electromagnetic waves by finitely many point-like obstacles

Chalons, C. \& Coquel, F., Modified Suliciu relaxation system and exact resolution of isolated shock waves

Chambolle, A., see Bellettini

Chertock, A., Kurganov, A., Polizzi, A. \& Timofeyev, I., Pedestrian flow models with slowdown interactions

Ciarlet, P. G. \& Mardare, C., Intrinsic formulation of the displacementtraction problem in linearized elasticity

Colombo, A., see Bassi

Conti, S. \& Dolzmann, G., Relaxation of a model energy for the cubic to tetragonal phase transformation in two dimensions

Coquel, F., Saleh, K. \& Seguin, N., A robust and entropy-satisfying numerical scheme for fluid flows in discontinuous nozzles

Coquel, F., see Chalons

Cots, O., see Bonnard

Couce-Calvo, J., see Casado-Díaz

Coudière, Y., Bourgault, Y. \& Rioux, M., Optimal monodomain approximations of the bidomain equations used in cardiac electrophysiology

Dalibard, A.-L., see Bonnivard

Dapogny, C., see Allaire

d'Avenia, P. \& Squassina, M., Soliton dynamics for the Schrödinger-Newton system

Davoli, E., Quasistatic evolution models for thin plates arising as low energy $\Gamma$-limits of finite plasticity

De Angelis, E., On the mathematical theory of post-Darwinian mutations, selection, and evolution

DeVore, R. \& Zuazua, E., Recovery of an initial

temperature from discrete sampling

24 (2014) 863

24 (2014) 937

24 (2014) 1091

24 (2014) 249

24 (2014) 1197

24 (2014) 1495

24 (2014) 2929

24 (2014) 2043

24 (2014) 937

24 (2014) 187

24 (2014) 1343

24 (2014) 1115

24 (2014) 2259

24 (2014) 2199

24 (2014) 553

24 (2014) 2085

24 (2014) 2723
Degond, P., Dimarco, G. \& Mac, T. B. N., Hydrodynamics of the KuramotoVicsek model of rotating self-propelled particles

Dimarco, G., see Degond

Dolfin, M. \& Lachowicz, M., Modeling altruism and selfishness in welfare dynamics: The role of nonlinear interactions

Dolzmann, G., see Conti

Donadello, C., see Andreianov

Dreyer, W., see Aki

Droniou, J., Finite volume schemes for diffusion equations: Introduction to and review of modern methods

Duchêne, V., Decoupled and unidirectional asymptotic models for the propagation of internal waves

Efendiev, Y., see Calo

Eftimie, R., see Buono

Egger, H. \& Schlottbom, M., Stationary radiative transfer with vanishing absorption

El Hajj, R. \& Méhats, F., Analysis of models for quantum transport of electrons in graphene layers

Etikyala, R., Göttlich, S., Klar, A. \& Tiwari, S., Particle methods for pedestrian flow models: From microscopic to nonlocal continuum models

Exl, L., Abert, C., Mauser, N. J., Schrefl, T., Stimming, H. P. \& Suess, D., FFT-based Kronecker product approximation to micromagnetic long-range interactions

Fabrizio, M., see Amendola

Fagnani, F., Fosson, S. M. \& Ravazzi, C., Consensuslike algorithms for estimation of Gaussian mixtures over large scale networks

Feischl, M., see Bruckner

Feng, Y.-H., Wang, S. \& Kawashima, S., Global existence and asymptotic decay of solutions to
24 (2014) 2487

24 (2014) 277

24 (2014) 277

24 (2014) 2361

24 (2014) 2929

24 (2014) 2685

24 (2014) 827

24 (2014) 1575

24 (2014) 1

24 (2014) 465

24 (2014) 327

24 (2014) 973

24 (2014) 2287

24 (2014) 2503

24 (2014) 1877

24 (2014) 1037

24 (2014) 381

24 (2014) 2627 
the non-isentropic EulerMaxwell system

Fosson, S. M., see Fagnani

Fried, E., see Seguin

Führer, T., see Bruckner

Galvis, J., see Calo

Georgoulis, E. H., see Cangiani

Gérard-Varet, D., see Bonnivard

Geymonat, G., Hendili, S., Krasucki, F. \& Vidrascu, M., Matched asymptotic expansion method for a homogenized interface model

Giesselmann, J., see Aki

Golden, J. M., see Amendola

Goldenits, P., see Bruckner

Goldman, M., see Bellettini

Goldstein, M., see Bissell

Göttlich, S., see Borsche

Göttlich, S., see Etikyala

Grasselli, M. \& Wu, H., Well-posedness and longtime behavior for the modified phase-field crystal equation

Gwiazda, P., see Carrillo

Gyrya, V., Lipnikov, K., Manzini, G. \& Svyatskiy, D., M-Adaptation in the mimetic finite difference method

Ha, S.-Y., Kang, M.-J. \& Kwon, B., A hydrodynamic model for the interaction of Cucker-Smale particles and incompressible fluid

Ha, S.-Y., see Li

Haberkorn, T., see Bergounioux

Hajaiej, H., Markowich, P. A. \& Trabelsi, S., Multiconfiguration Hartree-Fock theory for pseudorelativistic systems: The time-dependent case

Hendili, S., see Geymonat

Hoang, V. H. \& Schwab, C., $N$-term Wiener chaos approximation rates for elliptic PDEs with lognormal Gaussian random inputs

Hoang, V. H., see Xia

Houston, P., see Cangiani

$\mathrm{Hu}, \mathrm{G}$., see Challa
24 (2014) 2851

24 (2014) 381

24 (2014) 1729

24 (2014) 2627

24 (2014) 465

24 (2014) 2009

24 (2014) 2259

24 (2014) 573

24 (2014) 827

24 (2014) 1037

24 (2014) 2627

24 (2014) 1091

24 (2014) 719

24 (2014) 221

24 (2014) 2503

24 (2014) 2743

24 (2014) 2171

24 (2014) 1621

24 (2014) 2311

24 (2014) 1389

24 (2014) 2525

24 (2014) 599

24 (2014) 573

24 (2014) 797

24 (2014) 513

24 (2014) 2009

24 (2014) 863
Hughes, T. J. R., Amplitude-phase decompositions and the growth and decay of solutions of the incompressible Navier-Stokes and Euler equations

Jesslé, D., Novotný, A. \& Pokorný, M., Steady Navier-Stokes-Fourier system with slip boundary conditions

Joly, P., see Schmidt

Juntunen, M. \& Lee, J., A mesh-dependent norm analysis of low-order mixed finite element for elasticity with weakly symmetric stress

Kang, M.-J., see Ha

Kawashima, S., see Feng

Khalil, Z., see Bendahmane

Klar, A., see Borsche

Klar, A., see Borsche

Klar, A., see Etikyala

Knopoff, D., On a mathematical theory of complex systems on networks with application to opinion formation

Koumatos, K., see Ball

Krasucki, F., see Geymonat

Kraus, C., see Aki

Kühn, S., see Borsche

Kurganov, A., see Chertock

Kwon, B., see Ha

Lachowicz, M., see Dolfin

Larsen, C. J. \& Slastikov, V., Dynamic cohesive fracture: Models and analysis

Laurençot, Ph. \& Walker, Ch., A free boundary problem modeling electrostatic MEMS: II. Nonlinear bending effects

Lee, J., see Juntunen

Li, J., Li, T. \& Wang, Z.A., Stability of traveling waves of the Keller-Segel system with logarithmic sensitivity

$\mathrm{Li}, \mathrm{T}$., see $\mathrm{Li}$

Li, X. \& Scott, M. A., Analysis-suitable Tsplines: Characterization, refineability, and approximation

Li, Z., Ha, S.-Y. \& Xue, X., Emergent phenomena in an ensemble of
24 (2014) 1017

24 (2014) 751

24 (2014) 1823

24 (2014) 2155

24 (2014) 2311

24 (2014) 2851

24 (2014) 145

24 (2014) 221

24 (2014) 359

24 (2014) 2503

24 (2014) 405

24 (2014) 1937

24 (2014) 573

24 (2014) 827

24 (2014) 359

24 (2014) 249

24 (2014) 2311

24 (2014) 2361

24 (2014) 1857

24 (2014) 2549

24 (2014) 2155

24 (2014) 2819

24 (2014) 2819

24 (2014) 1141 
Cucker-Smale particles under joint rooted leadership

Lima, E. A. B. F., Oden, J. T. \& Almeida, R. C., A hybrid ten-species phasefield model of tumor growth

Lipnikov, K., see Gyrya

Long, C. C., see Takizawa

Lorenz, T. \& Surulescu, C., On a class of multiscale cancer cell migration models: Well-posedness in less regular function spaces

Mac, T. B. N., see Degond

Maestre, F., see CasadoDíaz

Manzini, G., Russo, A. \& Sukumar, N., New perspectives on polygonal and polyhedral finite element methods

Manzini, G., see Bellomo

Manzini, G., see Gyrya

Mardare, C., see Ciarlet

Marini, L. D., see Beirão da Veiga

Markowich, P. A., see Hajaiej

Marsden, A. L., see Takizawa

Martín Gómez, J. D., see Casado-Díaz

Mauser, N. J., see Exl

Méhats, F., see El Hajj

Melenk, J. M., see Aurada

Meurer, A., see Borsche

Minjeaud, S., see Boyer

Morales-Rodrigo, C. \& Tello, J. I., Global existence and asymptotic behavior of a tumor angiogenesis model with chemotaxis and haptotaxis

Nadin, G., see Bouin

Nieto, J., see Calvo

Nochetto, R. H., Salgado, A. J. \& Tomas, I., The Micropolar Navier-Stokes equations: A priori error analysis

Nochetto, R. H., Salgado, A. J. \& Walker, S. W., A diffuse interface model for electrowetting with moving contact lines

Novotný, A., see Jesslé

Oden, J. T., see Lima
24 (2014) 1389

24 (2014) 2569

24 (2014) 1621

24 (2014) 2437

24 (2014) 2383

24 (2014) 277

24 (2014) 1343

24 (2014) 1665

24 (2014) 1453

24 (2014) 1621

24 (2014) 1197

24 (2014) 1541

24 (2014) 599

24 (2014) 2437

24 (2014) 1343

24 (2014) 1877

24 (2014) 2287

24 (2014) 113

24 (2014) 359

24 (2014) 2885

24 (2014) 427

24 (2014) 1165

24 (2014) 495

24 (2014) 1237

24 (2014) 67

24 (2014) 751

24 (2014) 2569
Ohlberger, M., see Berninger

Page, M., see Bruckner

Panasenko, G. P. \& Stavre, R., Asymptotic analysis of a viscous fluid-thin plate interaction: Periodic flow

Pankratov, L., see Amaziane

Patera, A. T., see Yano

Paulino, G. H., see Talischi

Perthame, B., Tang, M. \& Vauchelet, N., Traveling wave solution of the HeleShaw model of tumor growth with nutrient

Piatnitski, A., see Amaziane

Pierre, C., see Bouyssier

Plouraboué, F., see Bouyssier

Pokorný, M., see Jesslé

Polizzi, A., see Chertock

Praetorius, D., see Aurada

Praetorius, D., see Bruckner

Prigozhin, L., see Barrett

Privat, Y., see Bergounioux

Ravazzi, C., see Fagnani

Raymond, N., Breaking a magnetic zero locus: Asymptotic analysis

Rioux, M., see Coudière

Riviere, B. \& Sardar, S., Penalty-free discontinuous Galerkin methods for incompressible NavierStokes equations

Rocca, E. \& Rossi, R., A degenerating PDE system for phase transitions and damage

Rosini, M. D., see Andreianov

Rossi, R., see Rocca

Rudnicki, R. \& Tiuryn, J., Size distribution of gene families in a genome

Ruggeri, M., see Bruckner

Russo, A., see Beirão da Veiga

Russo, A., see Manzini

Saad, M., see Bendahmane

Saleh, K., see Coquel

Salgado, A. J., see Nochetto

Salgado, A. J., see Nochetto

Sander, O., see Berninger

Sardar, S., see Riviere

Schillen, P., see Borsche

Schjodt, K., see Takizawa

Schlottbom, M., see Egger
24 (2014) 901

24 (2014) 2627

24 (2014) 1781

24 (2014) 1421

24 (2014) 1903

24 (2014) 1701

24 (2014) 2601

24 (2014) 1421

24 (2014) 627

24 (2014) 627

24 (2014) 751

24 (2014) 249

24 (2014) 113

24 (2014) 2627

24 (2014) 991

24 (2014) 2525

24 (2014) 381

24 (2014) 2785

24 (2014) 1115

24 (2014) 1217

24 (2014) 1265

24 (2014) 2685

24 (2014) 1265

24 (2014) 697

24 (2014) 2627

24 (2014) 1541

24 (2014) 1665

24 (2014) 145

24 (2014) 2043

24 (2014) 67

24 (2014) 1237

24 (2014) 901

24 (2014) 1217

24 (2014) 221

24 (2014) 2437

24 (2014) 973 
Schmidt, K., Thöns-Zueva, A. \& Joly, P., High-order asymptotic expansion for the acoustics in viscous gases close to rigid walls

Schrefl, T., see Exl

Schwab, C., see Hoang

Scott, M. A., see $\mathrm{Li}$

Seguin, B. \& Fried, E., Roughening it - evolving irregular domains and transport theorems

Seguin, N., see Coquel

Sini, M., see Challa

Slastikov, V., see Larsen

Smetana, K., see Berninger

Soler, J., see Calvo

Squassina, M., see d'Avenia

Stavre, R., see Panasenko

Stimming, H. P., see Exl

Straughan, B., see Bissell

Suess, D., see Bruckner

Suess, D., see Exl

Sukumar, N., see Manzini

Surulescu, C., see Lorenz

Svyatskiy, D., see Gyrya

Takizawa, K., Bazilevs, Y., Tezduyar, T. E., Long, C. C., Marsden, A. L. \& Schjodt, K., ST and ALE-VMS methods for patient-specific cardiovascular fluid mechanics modeling

Talischi, C. \& Paulino, G. H., Addressing integration error for polygonal finite elements through polynomial projections: A patch test connection

Tang, M., see Perthame
24 (2014) 1823

24 (2014) 1877

24 (2014) 797

24 (2014) 1141

24 (2014) 1729

24 (2014) 2043

24 (2014) 863

24 (2014) 1857

24 (2014) 901

24 (2014) 495

24 (2014) 553

24 (2014) 1781

24 (2014) 1877

24 (2014) 719

24 (2014) 2627

24 (2014) 1877

24 (2014) 1665

24 (2014) 2383

24 (2014) 1621

24 (2014) 2437

24 (2014) 1701 24 (2014) 2601
Tello, J. I., see MoralesRodrigo

Tezduyar, T. E., see Takizawa

Thöns-Zueva, A., see Schmidt

Timofeyev, I., see Chertock

Tiuryn, J., see Rudnicki

Tiwari, S., see Etikyala

Tomas, I., see Nochetto

Trabelsi, S., see Hajaiej

Ulikowska, A., see Carrillo

Urban, K., see Yano

Vásquez, M. O., see Calvo

Vauchelet, N., see Perthame

Verani, M., see Antonietti

Vidrascu, M., see Geymonat

Walker, Ch., see Laurençot

Walker, S. W., see Nochetto

Wang, S., see Feng

Wang, Z.-A., see $\mathrm{Li}$

Winther, R., see Arnold

Wu, H., see Grasselli

Xia, B.-X. \& Hoang, V. H., Best $N$-term GPC approximations for a class of stochastic linear elasticity equations

Xue, X., see $\mathrm{Li}$

Yano, M., Patera, A. T. \& Urban, K., A spacetime $\quad h p$-interpolationbased certified reduced basis method for Burgers' equation

Zampini, S., Dual-primal methods for the cardiac bidomain model

Zuazua, E., see DeVore
24 (2014) 427

24 (2014) 2437

24 (2014) 1823

24 (2014) 249

24 (2014) 697

24 (2014) 2503

24 (2014) 1237

24 (2014) 599

24 (2014) 2171

24 (2014) 1903

24 (2014) 495

24 (2014) 2601

24 (2014) 1457

24 (2014) 573

24 (2014) 2549

24 (2014) 67

24 (2014) 2851

24 (2014) 2819

24 (2014) 783

24 (2014) 2743

24 (2014) 513

24 (2014) 1389

24 (2014) 1903

24 (2014) 667

24 (2014) 2487 